The Egyptian International Journal of Engineering Sciences \& Technology, Vol 4, No 1 (2000)

\title{
Tiirbuient Boundary Layer Flow over S-Shaped Surface With Side V Parallel to the Stream
}

ma Hlawa

\begin{abstract}
TurhukiU boimdarv layers over s-shaped surface atlacbed to a verfical side wall were Experimeufally mvesfigafed. Measurements were performed over the s-shaped surface At three distinctive regions; the neighborhood of the corner; a distance approximately equal the thickness of the mixed bomidary layer, and far distance from the side wall. The effect of attaching the sidewall to the s-shaped surface is striking, as it prevents the flow along the corner from separation in adverse gradients. The mixed boundary layer fJow in the corner increases the skin friction coefficient. Beyond the reattachment of the free boundary layer, at far distance from the corner, the turbulent structure has become strongly disturbed by the prior separation and hence thickened the boundary layer
\end{abstract}

